scavenger, whereas, the specialised claws of Deinonychus suggest active predation on animals about its own size.

How do such problems affect the concept of dinosaur endothermy? They do not affect other arguments, such as Ostrom's contention that erect gaits may only be possible for endotherms, or Wheeler's recent comments (Nature 275, 441; 1978) on cooling of the central nervous system. Erythrosuchids need not have been insulated, but did have whatever condition is responsible for "endothermic' bone. The problem is that doubtful claims tend to discredit real evidence. But even so, if one follows Charig's rejection of predator-prey ratios, the only major line of evidence remaining is that from bone histology, which cannot be certainly attributed to endothermy rather than bulk.

I muist also support Thulborn's view that small size, a naked skin, and endothermic physiology seems a lethal combination for hatchlings. This need not mean that dinosaurs were simply 'good reptiles', but their nearest possible approach to true endothermy, if their young lacked insulation, will have been some combination of fairly high metabolic rates with poikilothermy, (until temperature could be stabilised by bulk in the larger animals). Perhaps this type of physiology would be feasible for typically large animals, evolved in warm Middle to Upper Triassic conditions, but it does not correspond to the fully developed endothermy of mammals and birds, in which external insulation allows high stable temperatures to be maintained in small animals.

\section{Long-range molecules}

\section{from Graham Richards}

VAN der Waals forces are normally thought of as attractive. They are the weak attractions which hold molecules together with a binding energy often following the sixth power of the intermolecular separation. In fact this is only true for spherical charge distributions. Attractions of the $c / r^{\circ}$ form are appropriate for interactions between rare gas atoms but in more complicated instances this may not be true even between atoms. Perhaps most striking is the fact that if one of the atoms is in a spherically symmetrical $S$ state and the other in an electronic state of $\mathbf{P}$ type then not only is the dispersion force of the form $c / r^{3}$ but

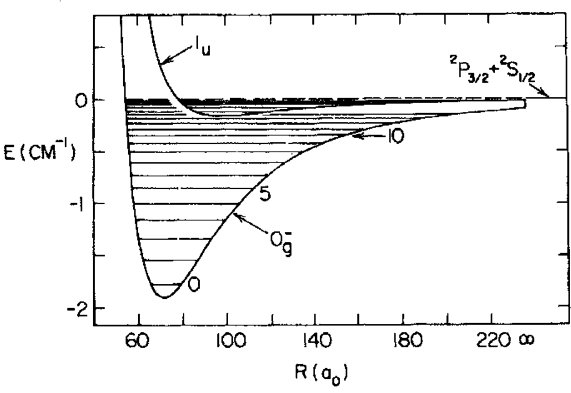

The potential-energy curves for two long range states of $\mathrm{Na}_{2}$.

it is repulsive!

This fact is utilised in a description of a new type of molecular electronic state by Stwally and Yea-Hwang Uang of the University of Iowa and Pichler of the Joint Institute for Laboratory Astrophysics in Boulder (Phys. Rev. Lett., 141, 1164; 1978).

The energy of a diatomic molecule is normally represented by a potential energy curve with a left-hand limit showing the vibrational turning point with the nuclei close together, and the right-hand branch the maximum stretch between the nuclei at the other extreme of vibration. Vibrational levels near dissociation give rise to 'long-range molecules' since although the molecule repeatedly vibrates classically from the inner turning point (typically $1 \AA$ separation) to the outer one (typically 10-100 $\AA$ ), it spends most of its time at the long range region.

The new type of pure long-range molecule is predicted to spend its entire time in long range regions with separations between $30 \AA$ and $100 \AA$. These long-range molecules will be possible for electronic states where the repulsive van der Waals forces at large internuclear separations result in there being a small bump in the potential energy curve and a very small well in which vibrations can take place. The local energy minima at very long range arise from the recoupling of angular momenta.

In the familiar type of diatomic molecule potential curve the range of internuclear separation shown is perhaps 1-10 $\AA$ with the depth of the potential well being several electron volts $\left(1 \mathrm{eV}\right.$ is approximately $8,000 \mathrm{~cm}^{-1}$ and $1,000 \mathrm{~cm}^{-1}$ is approximately $12 \mathrm{~kJ}$ $\mathrm{mol}^{-1}$ ). This may be contrasted with the curves drawn in the figure.

Although typical in shape the depths of the potential well are now less than $2 \mathrm{~cm}^{-1}$ and average separation is over $30 \AA$ (1 Bohr, $a_{0},=0.52917 \AA$ ). The

Graham Richards is a lecturer in the Physical Chemistry Laboratory, Oxford University. curves are those predicted by Stwalley et al. for two states of diatomic sodium.

Experimentally these states are visible as angular momentum recoupling extrema in the far wings of resonance lines of $R b$ and Cs. In particular, the red wing stellite on the $8521 \AA$ line of Cs corresponds to the $\mathrm{O}^{-}$s state illustrated in the figure for $\mathrm{Na}_{2}$. The authors hope to prepare molecular beams with significant populations of long-range levels of $\mathrm{Li}_{2}$ and $\mathrm{Na}_{2}$ and to observe rotational fine structure in bands involving the longrange states by laser spectroscopy.

\section{Metallic hydrogen}

from N. E. Cussack

A RECENT report by $P$. S. Hawke and fellow workers at the Lawrence Livermore Laboratory (Phys. Rev. Lett. 41, 994; 1978) that highly compressed hydrogen is a metal raises again one of the most interesting questions in the physics of condensed matter, namely, what decides whether a substance is metallic or not? Many theoretical and experimental studies, going back to classical papers by Mott in the $1950 \mathrm{~s}$, have made it clear that one decisive parameter is the density. In a way this is obvious; at room temperature liquid mercury, for example, is metallic but its vapour is not. But whether there is a critical density at which a sharp phase change from metal to non-metal occurs and, if so, what electronic mechanism is at work are still open to discussion and much depends on whether the material is crystalline, or structurally disordered as in a fluid.

For crystals, it holds that if the bands of allowed electron energy are either fully occupied or completely empty, then the material is nonmetallic. Partially empty bands permit metallic conductivity and therefore, if a full and an empty band can be made by a density change to overlap in energy, they will both become partially full and a metal will be created. The reverse could occur if the density change caused two overlapping energy bands to separate. These are called 'band crossing transitions' and are believed to occur in insulating iodine and metallic ytterbium which, under high pressures, reverse their roles and become a metal and an insulator, respectively. However, other considerations enter for monovalent metals like sodium which have half full energy bands. If these could be reduced in density-not possible in the crystallised 